\title{
Fermionic computation is non-local tomographic and violates monogamy of entanglement
}

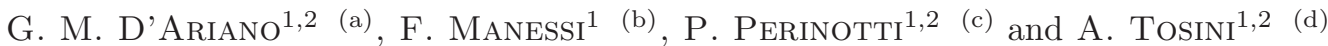 \\ 1 QUIT group, Dipartimento di Fisica, via Bassi 6, 27100 Pavia, Italy. \\ 2 INFN Sezione di Pavia, via Bassi, 6, 27100 Pavia, Italy.
}

PACS 03.67.Mn - Entanglement measures, witnesses, and other characterizations PACS 03.67.Lx - Quantum computation architectures and implementations

\begin{abstract}
We show that the computational model based on local Fermionic modes in place of qubits does not satisfy local tomography and monogamy of entanglement, and has mixed states with maximal entanglement of formation. These features directly follow from the parity superselection rule. We generalize quantum superselection rules to general probabilistic theories as sets of linear constraints on the convex set of states. We then provide a link between the cardinality of the superselection rule and the degree of holism of the resulting theory.
\end{abstract}

In his pioneering paper on physical computation [1, Feynman wondered about the possibility of simulating Fermions by local commuting quantum systems in interaction - what we would call nowadays a "quantum computer". Ever since, the relation between Fermions and local quantum systems has been largely investigated. The Jordan-Wigner map [2] transforms isomorphically the Fermionic algebra into a qubit algebra, and has been a valuable instrument for solving the $1 \mathrm{~d}$ XY spin-chains [3], or to extend to the Fermionic case notions as the entanglement [5], the entropic area law 6], and universal compu-tation [7, 8. However, in many applications one needs to map quantum algebras in an "isolocal" way, namely mapping local quantum operations into local ones, and nonlocal to nonlocal ones. The Jordan-Wigner transform is not isolocal, and this leads to some ambiguities in defining the partial trace 9 12, and in assessing the local nature of quantum operations [13. Here the Wigner superselection rule comes to help.

The Wigner superselection rule forbids superpositions between states with odd and even particle number, based on the simple argument of the impossibility of discriminating a $2 \pi$ rotation from the identity [14,15]. The Wigner superselection rule allows one to circumvent the problems connected to isolocality [5] without restoring it. The price

\footnotetext{
(a) E-mail: dariano@unipv.it

(b) E-mail: franco.manessi01@ateneopv.it

(c) E-mail: paolo.perinotti@unipv.it

(d) E-mail: alessandro.tosini@unipv.it
}

to pay, as we will see in this letter, is that the theory becomes non locally-tomographic [16, 17, namely one cannot discriminate between two nonlocal states using only local measurements, unlike quantum theory (QT).

The notion of local-tomography (also called localdiscriminability 18) has been introduced in the new context of general probabilistic theories, which has become the stage for the recent axiomatization program of QT. Examples of such theories are the classical information theory [17, the box-world [19, and the real quantum theory (RQT) 20,21. In such a framework, a theory that lacks local-tomography is called holistic 21. In this letter we will introduce a notion of superselection rule for a general probabilistic theory, corresponding to a linear constraint over the convex set of states. Such a notion contains the usual superselection rules of QT as special cases, but also includes other cases, e.g. RQT as a superselection restriction. We will provide a link between the number of linearly-independent constraints and the degree of holism of the resulting theory.

In addition to the feature of local-tomography, another characteristic trait of QT is the monogamy of entanglement, i.e. loosely speaking a limitation on the sharing of entanglement. For example, if two qubits are maximally entangled, neither of them can be entangled with any other system. After extending the usual notions of entanglement of formation and concurrence to the Fermionic scenario, we will show that in Fermionic quantum theory (FQT) entanglement is in general not monogamous, due 
to the Wigner superselection rule. As we will show, the monogamy violation goes hand in hand with the existence of maximally entangled states that are mixed. Moreover, one has Maximally-Entangled Sets (MES) 22 containing more than one bipartite state, whereas QT has only the singlet, and non-trivial MES's needs tripartite systems.

In the following we will restrict to probabilistic theories that are convex (i.e. all sets of transformations are convex) and causal [17] (namely, the probability of the preparation is independent of the choice of the observation test). Transformations include as special cases states and effects, and we will denote by $\operatorname{St}(\mathrm{A})$ and $\operatorname{Eff}(\mathrm{A})$ the convex set of (generally subnormalized) states and the convex set of effects of system A. In the presence of the non restriction hypothesis in its extended version (namely all admissible transformations belong to the theory) a theory is fully specified by the sets of states $\mathrm{St}(\mathrm{A})$ and by composition of systems. Imposing a superselection rule $\sigma$ on a theory corresponds to sectioning linearly all sets of transformations for each multipartite system. Under the non restriction hypothesis this reduces to sectioning linearly just the sets of states. Thus, superselecting the system A with the rule $\sigma$ means sectioning linearly $\mathrm{St}(\mathrm{A})$ giving a new set of states $\operatorname{St}(\overline{\mathrm{A}})$, which is identified with the system $\overline{\mathrm{A}}:=\sigma(\mathrm{A})$ of the constrained theory. For consistency, the superselection map $\sigma$ must commute with system composition, forcing the definition of composition for the constrained theory as $\sigma(\mathrm{A}) \sigma(\mathrm{B}):=\sigma(\mathrm{AB})$ (we remind that system composition is denoted by juxtaposition, namely the composed system of $\mathrm{A}$ and $\mathrm{B}$ is $\mathrm{AB}$ ). Notice that, being linear $\sigma$ preserves convexity of the theory. This means that e.g. in a QT with a superselection rule states from different sectors cannot be superimposed, but can be mixed.

In the following we will denote by $\mathrm{X}_{\mathbb{R}}$ the linear span of the set $\mathrm{X}$, e.g. $\operatorname{Eff}_{\mathbb{R}}(\mathrm{A})$ is just the set of linear functionals on states. The superselection rule $\sigma$ will be defined for an arbitrary system A through linearly independent effects $s_{i}^{\sigma} \in \operatorname{Eff}_{\mathbb{R}}(\mathrm{A}), i=1, \ldots V_{\mathrm{A}}^{\sigma}$ as follows

$$
\mathrm{St}[\sigma(\mathrm{A})]:=\left\{\rho \in \mathrm{St}(\mathrm{A}) \mid s_{i}^{\sigma}(\rho)=0, i=1, \ldots, V_{\mathrm{A}}^{\sigma}\right\} .
$$

Clearly $\operatorname{St}(\overline{\mathrm{A}}) \subseteq \operatorname{St}(\mathrm{A})$ and $\operatorname{Eff}(\overline{\mathrm{A}}) \subseteq \operatorname{Eff}(\mathrm{A})$. One has

$$
D_{\overline{\mathrm{A}}}=D_{\mathrm{A}}-V_{\mathrm{A}}^{\sigma},
$$

where $D_{\mathrm{A}}:=\operatorname{dim}\left[\operatorname{St}_{\mathbb{R}}(\mathrm{A})\right]$. For a general theory one has $D_{\mathrm{AB}} \geq D_{\mathrm{A}} D_{\mathrm{B}}$, and this provides an upper bound for the number of independent constraints of a composite system, i.e. $V_{\mathrm{AB}}^{\sigma} \leq D_{\mathrm{A}} V_{\mathrm{B}}^{\sigma}+D_{\mathrm{B}} V_{\mathrm{A}}^{\sigma}+D_{\mathrm{AB}}-V_{\mathrm{A}}^{\sigma} V_{\mathrm{B}}^{\sigma}$.

It is easy to see that for any $b \in \operatorname{Eff}(\overline{\mathrm{B}})$ and any $i=$ $1, \ldots, V_{\mathrm{A}}^{\sigma}$, the functional $s_{i}^{\sigma} \otimes b \in \operatorname{Eff}_{\mathbb{R}}(\mathrm{AB})$ is a constraint for $\overline{\mathrm{AB}}$. Indeed, suppose by contradiction that $s_{i}^{\sigma} \otimes b(\rho) \neq$ 0 for $\rho \in \operatorname{St}(\overline{\mathrm{AB}})$, then since $b(\rho)=\alpha$ is a valid state for $\overline{\mathrm{A}}$, we have $s_{i}^{\sigma}(\alpha) \neq 0$ against the hypothesis. The same argument holds exchanging the subsystems $\mathrm{A}$ and $\mathrm{B}$, and we conclude that the composite system $\overline{\mathrm{AB}}$ has at least $D_{\overline{\mathrm{A}}} V_{\mathrm{B}}^{\sigma}+D_{\overline{\mathrm{B}}} V_{\mathrm{A}}^{\sigma}=D_{\mathrm{A}} V_{\mathrm{B}}^{\sigma}+D_{\mathrm{B}} V_{\mathrm{A}}^{\sigma}-2 V_{\mathrm{A}}^{\sigma} V_{\mathrm{B}}^{\sigma}$ of linearly independent constraints. In summary we have the bounds

$$
\begin{aligned}
& V_{\mathrm{AB}}^{\sigma} \geq D_{\mathrm{A}} V_{\mathrm{B}}^{\sigma}+D_{\mathrm{B}} V_{\mathrm{A}}^{\sigma}-2 V_{\mathrm{A}}^{\sigma} V_{\mathrm{B}}^{\sigma}, \\
& V_{\mathrm{AB}}^{\sigma} \leq D_{\mathrm{A}} V_{\mathrm{B}}^{\sigma}+D_{\mathrm{B}} V_{\mathrm{A}}^{\sigma}-V_{\mathrm{A}}^{\sigma} V_{\mathrm{B}}^{\sigma}+D_{\mathrm{AB}}-D_{\mathrm{A}} D_{\mathrm{B}} .
\end{aligned}
$$

A superselected theory saturating the lower bound in Eq. (2) is called minimal. For such a theory the constraints for bipartite systems are only those of the form $s_{i}^{\sigma} \otimes b$ and $a \otimes s_{j}^{\sigma}$, with $a \in \operatorname{Eff}(\overline{\mathrm{A}})$ and $b \in \operatorname{Eff}(\overline{\mathrm{B}})$. A minimal superselected theory can be built "bottom-up" by defining the constraints on the elementary systems.

Before proceeding, we recall the notions of $n$-local effect and of $n$-local-tomographic theory 21. We call an effect $n$-local if it can be written as conical combination of composite effects made of effects that are at most $n$-partite. A set of effects $\mathrm{E}$ is called separating for a set of states $\mathrm{S}$ if any two states of $\mathrm{S}$ are discriminated by an effect of $\mathrm{E}$. We call a theory $n$-local-tomographic if the set of $n$-local effects is separating for multipartite states. For a localtomographic theory (i.e. $n=1$ ) one has $D_{\mathrm{AB}}=D_{\mathrm{A}} D_{\mathrm{B}}$. Notice that an $n$-local-tomographic theory is also $(n+1)$ local-tomographic. We will call a theory strictly $n$-localtomographic if it is $n$-local-tomographic but not $(n-1)$ local-tomographic.

By definition, a strictly bilocal-tomographic theory (i.e. $n=2$ ) has 21 .

$$
\begin{aligned}
& D_{\mathrm{AB}}>D_{\mathrm{A}} D_{\mathrm{B}}, \\
& D_{\mathrm{ABC}} \leq D_{\mathrm{A}} D_{\mathrm{B}} D_{\mathrm{C}}+\widetilde{D}_{\mathrm{AB}} D_{\mathrm{C}}+\widetilde{D}_{\mathrm{BC}} D_{\mathrm{A}}+\widetilde{D}_{\mathrm{CA}} D_{\mathrm{B}},
\end{aligned}
$$

where

$$
\widetilde{D}_{\mathrm{AB}}:=D_{\mathrm{AB}}-D_{\mathrm{A}} D_{\mathrm{B}} .
$$

A strictly bilocal-tomographic theory that saturates the upper bound will be called maximally bilocal-tomographic, and it requires all 2-local effects to separate multipartite states.

In the following we will focus on the superselection of a local-tomographic theory. This is the case, for example, of QT with parity or charge-superselection and of the RQT. In this case $D_{\mathrm{AB}}=D_{\mathrm{A}} D_{\mathrm{B}}$ and therefore Eq. (2) becomes

$$
\begin{aligned}
& V_{\mathrm{AB}}^{\sigma} \geq D_{\mathrm{A}} V_{\mathrm{B}}^{\sigma}+D_{\mathrm{B}} V_{\mathrm{A}}^{\sigma}-2 V_{\mathrm{A}}^{\sigma} V_{\mathrm{B}}^{\sigma}, \\
& V_{\mathrm{AB}}^{\sigma} \leq D_{\mathrm{A}} V_{\mathrm{B}}^{\sigma}+D_{\mathrm{B}} V_{\mathrm{A}}^{\sigma}-V_{\mathrm{A}}^{\sigma} V_{\mathrm{B}}^{\sigma} .
\end{aligned}
$$

In this scenario we have a striking relation between the discriminability of states and superselection rules. Indeed a minimal superselected theory is maximally bilocaltomographic. This can be proved by evaluating $D \overline{\mathrm{ABC}}$ using the saturated bound of Eq. (6) and the identities of Eq. (5), Eq. (19), and $\overline{\mathrm{ABC}}=\overline{\mathrm{A}}(\overline{\mathrm{BC}})$, and noticing that it is equal to the RHS of Eq. (4). While a minimal superselected theory is maximally bilocal-tomographic, a theory saturating the upper bound (7) is local-tomographic. In the intermediate range one can find superselected theories of any degree of holism.

We now give a precise definition of the FQT. The FQT is the theory with no-restriction hypothesis, whose 
generic system $\mathrm{N}_{\mathrm{F}}$ is the quantum one made only of a finite number $N$ of qubits, satisfying the constraint that all states must commute with the parity operator $P_{N}:=\frac{1}{2}\left(I+\prod_{j=1}^{N} \sigma_{z}^{(j)}\right)\left(\sigma_{\alpha}\right.$ denotes a Pauli matrix $)$. The system $1_{\mathrm{F}}$ corresponds to the so-called local Fermionic mode (LFM), and the state spaces introduced here are the same as in Bravyi and Kitaev [7, however, the norestriction hypothesis allows for more transformations in our case. Locality of transformations, necessary for defining the composition of systems, is given in terms of the local Fermionic algebra, which is generated by the creation and annihilation operators $\psi_{i}^{\dagger}$ and $\psi_{i}$, respectively, with $i=1, \ldots N$, satisfying the anti-commutation relations $\left[\psi_{i}^{\dagger}, \psi_{j}\right]_{+}=\delta_{i j} I,\left[\psi_{i}, \psi_{j}\right]_{+}=0$. Let $|\Omega\rangle$ be the unique joint eigenvector of the operators $\psi_{j}^{\dagger} \psi_{j}$ with zero eigenvalue, and build a representation of field operators for a given ordering $1,2, \ldots N$ of the qubits, given by the orthonormal basis for $\mathbb{C}^{2^{N}}$ :

$$
\left|q_{1}, \ldots, q_{N}\right\rangle:=\psi_{1}^{\dagger q_{1}} \ldots \psi_{N}^{\dagger q_{N}}|\Omega\rangle \quad q_{i}=0,1 ; i=1, \ldots, N
$$

where $\left|q_{1}, \ldots, q_{N}\right\rangle$ are the joint eigenvectors for the qubit $\sigma_{z}^{(j)}$ for $j=1, \ldots, N$, forming a basis for the Fock space. Notice that a vector of Eq. (8) corresponds to a Slater determinant in the first quantization formalism. The chosen ordering identifies a specific Jordan-Wigner transform. We now can define locality of transformations. We say that an admissimble transformation of the $\mathrm{N}_{\mathrm{F}}$ system is local on the subsystem $\mathrm{M}_{\mathrm{F}}$ with $M<N$ if the Kraus operators belong the representation of the field algebra of $\mathrm{M}_{\mathrm{F}}$. The parity super-selection rule forbids superpositions of vectors belonging to $\mathcal{H}_{0}$ and $\mathcal{H}_{1}$ eigen-spaces of the parity operators in $\mathbb{C}^{2^{N}}$, and splits the operator spaces representing $\operatorname{Eff}_{\mathbb{R}}\left(\mathrm{N}_{\mathrm{F}}\right)=\operatorname{St}_{\mathbb{R}}\left(\mathrm{N}_{\mathrm{F}}\right)$ as $\operatorname{Herm}\left(\mathcal{H}_{0}\right) \oplus \operatorname{Herm}\left(\mathcal{H}_{1}\right)$, whose operators are spanned by products of even numbers of field operators.

In the following we will denote by $\mathrm{N}_{\mathrm{Q}}$ the multipartite system of $N$ qubits, with $2^{N}$-dimensional Hilbert space. Since QT is local-tomographic we have $D_{\mathrm{N}_{\mathrm{Q}}}=D_{1_{\mathrm{Q}}}^{N}=$ $2^{2 N}$. On the other hand, according to the parity prescription the dimension of the Fermionic system $\mathrm{N}_{\mathrm{F}}$ is $D_{\mathrm{N}_{\mathrm{F}}}=2^{2 N-1}=D_{\mathrm{N}_{\mathrm{Q}}} / 2$. Notice that the single-LFM system $1_{\mathrm{F}}$ has only two possible pure states $|0\rangle,|1\rangle$, thus corresponding to the classical bit, whereas the linear space of states for the system of $N$ LFM's is $\operatorname{St}_{\mathbb{R}}\left(\mathrm{N}_{\mathrm{F}}\right)=\operatorname{Eff}_{\mathbb{R}}\left(\mathrm{N}_{\mathrm{F}}\right)=$ $\operatorname{Herm}\left(\mathbb{C}^{2^{N-1}}\right) \oplus \operatorname{Herm}\left(\mathbb{C}^{2^{N-1}}\right)$, namely the direct sum of two copies of the state-space of $N-1$ qubits.

The FQT saturates the bound of Eq. (4), and is then maximally bilocal-tomographic. Indeed, for elementary Fermionic systems we have

$$
8=D_{2_{\mathrm{F}}}>D_{1_{\mathrm{F}}}^{2}=4, \quad D_{3_{\mathrm{F}}}=D_{1_{\mathrm{F}}}^{3}+3 \widetilde{D}_{2_{\mathrm{F}}} D_{1_{\mathrm{F}}}=32
$$

where $\widetilde{D}_{2_{\mathrm{F}}}=D_{2_{\mathrm{F}}}-D_{1_{\mathrm{F}}}^{2}=4$ is the dimension of the non-local component of $2_{\mathrm{F}}$. The full theory is maximally bilocal-tomographic, indeed, the number of independent local and 2-local effects for $N$ LFM's is $\sum_{k=0}^{\lfloor n / 2\rfloor}\left(\begin{array}{c}n \\ 2 k\end{array}\right) D_{1_{\mathrm{F}}}^{n-2 k} \widetilde{D}_{2_{\mathrm{F}}}^{k}=2^{2 n-1}=D_{\mathrm{N}_{\mathrm{F}}}$. We emphasize that the FQT provides an example of a bilocal-tomographic theory whose systems do not satisfy the dimensional prescription in Ref. 21] (see note1).

Besides being bilocal-tomographic, the FQT is also a minimally superselected QT of qubits. It is easy to see that the $1_{F}$ system can be achieved from the qubit by means of the superselection constraints $\operatorname{Tr}\left[\sigma_{x} \rho\right]=\operatorname{Tr}\left[\sigma_{y} \rho\right]=0$ for all $\rho \in \operatorname{St}\left(1_{\mathrm{F}}\right)$, hence $D_{1_{\mathrm{F}}}=D_{1_{\mathrm{Q}}}-V_{1_{\mathrm{Q}}}^{\sigma}$ with $V_{1_{\mathrm{Q}}}^{\sigma}=2$. The whole FQT can be built bottom-up by minimally extending the constraints to the composite systems. Indeed the lower bound in Eq. (7) is achieved.

Since the FQT is minimally superselected from a local-tomographic theory, it must be maximally bilocaltomographic. This is indeed the case, as one can see from the dimensional analysis in Eq. (91).

It is worth mentioning that the FQT is not the only minimal superselected QT. Another example is given by RQT. Its systems $\mathrm{N}_{\mathrm{R}}$ have dimensions $D_{\mathrm{N}_{\mathrm{R}}}=d_{\mathrm{N}_{\mathrm{R}}}\left(d_{\mathrm{N}_{\mathrm{R}}}+\right.$ 1)/2 with $d_{N_{R}}$ the number of perfectly distinguishable states for the system $\mathrm{N}_{\mathrm{R}}$. On the other hand one has $\mathrm{N}_{\mathrm{R}}=\sigma\left(\mathrm{N}_{\mathrm{Q}}\right)$ where the superselection rule is given by the constraint $\rho-\rho^{T}=0$, with $T$ denoting transposition with respect to a fixed basis taken as real, that for $1_{\mathrm{R}}$ (one rebit) corresponds to the linear constraint $\operatorname{Tr}\left[\sigma_{y} \rho\right]=0$. The RQT is minimally superselected, since the number of constraints for the composite system $\mathrm{N}_{\mathrm{R}} \mathrm{M}_{R}$ given by $V_{\mathrm{N}_{\mathrm{R}} \mathrm{M}_{R}}^{\sigma}=\frac{1}{2} d_{\mathrm{N}_{\mathrm{R}}} d_{\mathrm{M}_{R}}\left(d_{\mathrm{N}_{\mathrm{R}}} d_{\mathrm{M}_{R}}-1\right)$ saturates the lower bound (7). Then the theory is maximally bilocaltomographic, as pointed out in [21].

Notice that, due to the parity constraint, the FQT retains only superpositions of pure states with total occupation numbers that are equal modulo 2. If instead we allow only superpositions with total occupation numbers that are equal modulo $k$ for any integer $k$, we get a theory that is $k$-local-tomographic.

We now study entanglement in the FQT, and show that it shares some features with the RQT, as the existence of maximally-entangled mixed states, and the violation of entanglement monogamy. We will see that these phenomena are due to the fact that both theories are superselected versions of QT. One would conjecture that both features may be related to the non local-tomographic nature of the theories, however, this remains an open issue.

In a general probabilistic theory entanglement must be quantified in operational terms, namely as a resource for performing a task. For example, entanglement in QT represents the resource needed to prepare states of the theory under the restriction of LOCC (local operations and

${ }^{1}$ In Ref. 21] the authors after proving that $D_{\mathrm{AB}}-D_{\mathrm{A}} D_{\mathrm{B}}=$ $L_{\mathrm{A}} L_{\mathrm{B}}$ for some integers $L_{\mathrm{A}}$ and $L_{\mathrm{B}}$, under the assumption that $D_{\mathrm{A}}+L_{\mathrm{A}}, D_{\mathrm{A}}-L_{\mathrm{A}}$ are strictly increasing functions of the number of perfectly discriminable states $d_{\mathrm{A}}$, they prove that in a bilocaltomographic theory one has $D_{\mathrm{A}}=\frac{1}{2}\left(d_{\mathrm{A}}^{r}+d_{\mathrm{A}}^{s}\right)$ for some integers $r, s$ satisfying $r \geq s>0$. The strict monotonicity of the function $D_{\mathrm{A}}-L_{\mathrm{A}}$ is too restrictive and it excludes the FQT from the set of admissible bilocal-tomographic theories. Indeed, for the FQT we have $D_{\mathrm{N}_{\mathrm{F}}}-L_{\mathrm{N}_{\mathrm{F}}}=0$ for any $\mathrm{N}_{\mathrm{F}}$. 
classical communication). For bipartite states in QT all measures of entanglement refer to a standard unit - the ebit - which is the amount of entanglement of a bipartite singlet state, and the so-called entanglement of formation is the number of ebits that are needed to achieve the state by LOCC. Since the FQT is non-classical, considering entanglement as a resource under LOCC is meaningful. A full theory of entanglement for the FQT would require a complete analysis of the transformations of states under LOCC: this is beyond the scope of the present letter. However, here we will show that, independently of such analysis, one can assess features that are very different from those of entanglement in QT. These are: 1) the existence of mixed states with maximal entanglement of formation; 2) the need of MES 22 for bipartite states; 3) bipartite states with maximal entanglement of formation that do not belong to a MES; 4) the violation of monogamy of entanglement.

We now extend the notion of concurrence [23] and provide a lower bound to entanglement of formation [24] for the FQT.

In the usual quantum scenario, the entanglement of formation is defined for a generally mixed state $\rho \in \operatorname{St}(\mathrm{AB})$ as follows $E(\rho):=\min _{\mathcal{D}_{\rho}} \sum_{i} p_{i} S\left(\operatorname{Tr}_{\mathrm{A}}\left|\Psi_{i}\right\rangle\left\langle\Psi_{i}\right|\right)$, where $S(\sigma)$ is the von Neumann entropy of the state $\sigma$, and $\left.\mathcal{D}_{\rho}:=\left\{\left\{p_{i},\left|\Psi_{i}\right\rangle\right\}\left|\rho=\sum_{i} p_{i}\right| \Psi_{i}\right\rangle\left\langle\Psi_{i}\right|\right\}$ is the set of all the pure decomposition of the mixed state $\rho$. One has $E(\rho)=\lim _{n \rightarrow \infty} F_{n}(\rho) / n$, where $F_{n}(\rho)$ is the minimum number of singlets states needed by two parties in order to prepare via LOCC $n$ random states $\left|\Psi_{i}\right\rangle$ in any decomposition that achieves $E(\rho)[25$. The bound is achieved for pure states $[26$. For a mixed state $\rho$ of two qubits one has $E(\rho)=\mathcal{E}(C(\rho))$, with $\mathcal{E}(x):=h\left(\frac{1+\sqrt{1-x^{2}}}{2}\right), h$ the binary Shannon entropy, and the concurrence $C(\rho)$ defined as

$$
C(\rho):=\min _{\mathcal{D}_{\rho}} \sum_{i} p_{i} C\left(\left|\Psi_{i}\right\rangle\right),
$$

with $C(|\Psi\rangle)$ for pure states given in Ref. 23. Both the entanglement of formation and the concurrence are zero if and only if the state $\rho$ is separable, and for two qubits they reach the maximum value 1 if and only if $\rho$ is a maximally entangled state.

In Ref. 27, both the entanglement of formation and the concurrence have been specialized to RQT restricting the minimum to the set of pure decompositions $\mathcal{D}_{\rho}^{\mathrm{R}}$ on real states. In Ref. 5 the entanglement of formation has been extended to the FQT; here we do the same for the concurrence

$$
\begin{aligned}
& E_{\mathrm{F}}(\rho):=\min _{\mathcal{D}_{\rho}^{\mathrm{F}}} \sum_{i} p_{i} E\left(\left|\Psi_{i}\right\rangle\right), \\
& C_{\mathrm{F}}(\rho):=\min _{\mathcal{D}_{\rho}^{\mathrm{F}}} \sum_{i} p_{i} C\left(\left|\Psi_{i}\right\rangle\right),
\end{aligned}
$$

with $\mathcal{D}_{\rho}^{\mathrm{F}}$ the set of all the pure decompositions of $\rho$ that satisfy the parity superselection rule. Since each mixed state is parity-decomposed uniquely as $\rho=p_{0} \rho_{0}+p_{1} \rho_{1}$ and all Fermionic decompositions in $\mathcal{D}_{\rho}^{\mathrm{F}}$ must preserve $p_{0}$ and $p_{1}$, one has $E_{\mathrm{F}}(\rho)=p_{0} E_{\mathrm{F}}\left(\rho_{0}\right)+p_{1} E_{\mathrm{F}}\left(\rho_{1}\right)$ and $C_{\mathrm{F}}(\rho)=$ $p_{0} C_{\mathrm{F}}\left(\rho_{0}\right)+p_{1} C_{\mathrm{F}}\left(\rho_{1}\right)$. Moreover, since $\mathcal{D}_{\rho_{i}}^{\mathrm{F}} \equiv \mathcal{D}_{\rho_{i}}$, we have $E_{\mathrm{F}}\left(\rho_{i}\right)=E\left(\rho_{i}\right)$ and $C_{\mathrm{F}}\left(\rho_{i}\right)=C\left(\rho_{i}\right)$, hence

$$
\begin{aligned}
& E_{\mathrm{F}}(\rho)=p_{0} E\left(\rho_{0}\right)+p_{1} E\left(\rho_{1}\right), \\
& C_{\mathrm{F}}(\rho)=p_{0} C\left(\rho_{0}\right)+p_{1} C\left(\rho_{1}\right) .
\end{aligned}
$$

The above definition of entanglement of formation is not proved to have the same operational asymptotic interpretation as in QT, however, one can prove that it is a lower bound for it, since bipartite fermionic LOCC's are all admissible quantum LOCC's, and any fermionic entangled resource-state has a quantum entanglement of formation smaller than (or equal to) one. Notice that, unlike QT 23 and RQT 27, the quantities $E_{\mathrm{F}}$ and $C_{\mathrm{F}}$ do not satisfy the relation $E_{\mathrm{F}}(\rho)=\mathcal{E}\left(C_{\mathrm{F}}(\rho)\right)$. Nevertheless we have that $E_{\mathrm{F}}(\rho) \geq \mathcal{E}\left(C_{\mathrm{F}}(\rho)\right)$, and for a maximally-entangled state $\Phi$ it is $E_{\mathrm{F}}(\Phi)=\mathcal{E}\left(C_{\mathrm{F}}(\Phi)\right)=1$. Therefore, when $E_{F}(\rho)=1, E_{F}$ coincides with the operational entanglement of formation. Moreover, notice that the states of Eq. (8) have Fermionic entanglement of formation equal to zero, according to the fact that a single Slater determinant in the Fermionic theory is actually a product state.

Using the quantities $E_{\mathrm{F}}$ and $C_{\mathrm{F}}$ we can show that in the FQT there exist maximally-entangled mixed states. The state

$$
\Phi:=\frac{1}{4}\left(I \otimes I+\sigma_{x} \otimes \sigma_{x}\right),
$$

is the equal mixture of the Fermionic pure states $\left|\Psi_{0}\right\rangle=$ $\frac{1}{\sqrt{2}}(|00\rangle+|11\rangle)$ and $\left|\Psi_{1}\right\rangle=\frac{1}{\sqrt{2}}(|01\rangle+|10\rangle)$. It is easy to check that $E_{\mathrm{F}}(\Phi)=C_{\mathrm{F}}(\Phi)=1$, i.e. $\Phi$ has maximal entanglement of formation. On the other hand in QT $\Phi$ is separable since it can be regarded as the equal mixture of the pure states $|+\rangle|+\rangle,|-\rangle|-\rangle$, with $| \pm\rangle=\frac{1}{\sqrt{2}}(|0\rangle \pm|1\rangle)$, which gives $E(\Phi)=C(\Phi)=0$. Such a decomposition, however, is not allowed in the Fermionic case, because the states $| \pm\rangle$ violate the parity superselection rule. We could have replaced $\sigma_{x}$ in Eq. (15) with any linear combination of $\sigma_{x}$ and $\sigma_{y}$ according to the superselection constraints $\operatorname{Tr}\left[\sigma_{x} \rho\right]=\operatorname{Tr}\left[\sigma_{y} \rho\right]=0$ on the single LFM system. Since in RQT we have only the linear constraint $\operatorname{Tr}\left[\sigma_{y} \rho\right]=0$ for one rebit, the same argument holds for the state in Eq. (15) with $\sigma_{x}$ replaced by $\sigma_{y}$ [27, namely the theory has mixed maximally-entangled states.

As already mentioned, the state $\Phi$ despite having maximum entanglement of formation, cannot be transformed by LOCC into a maximally-entangled pure state. It actually happens that for two LFM's the concept of maximallyentangled state under LOCC has to be superseded by the concept of MES, as it has already been pointed out for $n$ partite quantum entanglement with $n \geq 3$ [22. A MEs for an $n$-partite system is the minimal set of $n$-partite states such that any other $n$-partite state can be obtained by LOCC from a state in the set. Two examples of MES for two LFM's are the set of all even non-factorized pure states with positive coefficients, and the set of all odd non factor- 
ized pure states (notice that LOCC can change the parity using the map $\left.\sigma_{x} \cdot \sigma_{x}\right)$.

Consider now the $3_{\mathrm{F}}$ pure state $\left|\Phi_{p}\right\rangle:=\frac{1}{2}(|000\rangle+|110\rangle+$ $|011\rangle+|101\rangle)$. If we trace the state $\left|\Phi_{p}\right\rangle$ over any one of the three LFM we find that the reduced bipartite state of two LFM's is the mixed state $\Phi$ of Eq. (15) which has maximal entanglement of formation. Therefore, in the FQT, as well as in RQT [28, monogamy of entanglement is violated, since the amount of entanglement can be totally shared by each pair of systems, a feature forbidden in QT.

We conclude this letter by observing that, while Fermionic computation and standard quantum computation have been shown to be equivalent [7, our findings about Fermionic entanglement suggests that the same may not hold for distributed Fermionic computation.

$$
* * *
$$

This work has been supported in part by the Templeton Foundation under the project ID\# 43796 A QuantumDigital Universe.

\section{REFERENCES}

[1] Feynman R., International journal of theoretical physics, 21 (1982) 467.

[2] Jordan P. and Wigner E., Zeitschrift für Physik, 47 (1928) 631.

[3] Onsager L., Physical Review, 65 (1944) 117.

[4] Lieb E., Schultz T. and Mattis D., Rev. Mod. Phys, 36 (1964) 856

[5] Bañuls M.-C., Cirac J. I. and Wolf M. M., Phys. Rev. A, 76 (2007) 022311.

[6] Wolf M. M., Phys. Rev. Lett., 96 (2006) 010404.

[7] Bravyi S. B. and Kitaev A. Y., Annals of Physics, 298 (2002) 210.

[8] Pineda C., Barthel T. and Eisert J., Phys. Rev. A, 81 (2010) 050303.

[9] Montero M. and Martín-Martínez E., Phys. Rev. A, 83 (2011) 062323

[10] Brádler K. and Jáuregui R., Phys. Rev. A, 85 (2012) 016301.

[11] Montero M. and Martín-Martínez E., Phys. Rev. A, 85 (2012) 016302

[12] Frits N., Lee A. R. and Bruschi D. E., Phys. Rev. A, 87 (2013) 022338.

[13] Verstraete F. and Cirac J., Journal of Statistical Mechanics: Theory and Experiment, 2005 (2005) P09012.

[14] Streater R. F. and Wightman A. S., PCT, spin and statistics, and all that (Princeton University Press) 1964.

[15] Weinberg S., The quantum theory of fields: Foundations. Vol. 1 (Cambridge university press) 2005.

[16] D'Ariano G. M., Probabilistic theories: What is special about quantum mechanics? in Philosophy of Quantum Information and Entanglement, edited by BoKULICH A. and JAeger G., (Cambridge University Press.) 2010 Ch. 5.

[17] Chiribella G., D'Ariano G. M. and Perinotti P., Phys. Rev. A, 84 (2011) 012311.

[18] Chiribella G., D'Ariano G. M. and Perinotti P., Phys. Rev. A, 81 (2010) 062348.
[19] Barrett J., Phys. Rev. A, 75 (2007) 032304. http://link.aps.org/doi/10.1103/PhysRevA.75.032304

[20] Stueckelberg E. and Guenin M., Helv. Phys. Acta, 34 (1961) 621.

[21] Hardy L. and Wootters W. K., Foundations of Physics, 42 (2012) 454.

[22] De Vicente J. I., Spee C. and Kraus B., Phys. Rev. Lett., 111 (2013) 110502.

[23] Hill S. and Wootters W. K., Physical review letters, 78 (1997) 5022.

[24] Bennett C. H., DiVincenzo D. P., Smolin J. A. and Wootters W. K., Physical Review A, 54 (1996) 3824.

[25] Wootters W. K., Quantum Information 85 Computation, 1 (2001) 27.

[26] Popescu S. and Rohrlich D., Phys. Rev. A, 56 (1997) R3319.

[27] Caves C. M., Fuchs C. A. and Rungta P., Foundations of Physics Letters, 14 (2001) 199.

[28] Wootters W., Foundations of Physics, 42 (2012) 19. http://dx.doi.org/10.1007/s10701-010-9488-1 